\title{
Utilization of alternate chirality enantiomers in microbial communities
}

\author{
Elena V. Pikuta* and Richard B. Hoover \\ NSSTC/NASA/Astrobiology Laboratory \\ 320 Sparkman Drive, Huntsville 35805 AL USA \\ *evpikuta@gmail.com; phone: +1 (256) 961-7868
}

\begin{abstract}
Our previous study of chirality led to interesting findings for some anaerobic extremophiles: the ability to metabolize substrates with alternate chirality enantiomers of amino acids and sugars. We have subsequently found that not just separate microbial species or strains but entire microbial communities have this ability. The functional division within a microbial community on proteo- and sugarlytic links was also reflected in a microbial diet with $L$ sugars and $D$-amino acids.

Several questions are addressed in this paper. Why and when was this feature developed in a microbial world? Was it a secondary de novo adaptation in a bacterial world? Or is this a piece of genetic information that has been left in modern genomes as an atavism? Is it limited exclusively to prokaryotes, or does this ability also occur in eukaryotes? In this article, we have used a broader approach to study this phenomenon using anaerobic extremophilic strains from our laboratory collection. A series of experiments were performed on physiologically different groups of extremophilic anaerobes (pure and enrichment cultures). The following characteristics were studied: 1) the ability to grow on alternate chirality enantiomers - $L$-sugars and $D$ - amino acids; 2) Growthinhibitory effect of alternate chirality enantiomers; 3) Stickland reaction with alternate chirality amino acids. The results of this research are presented in this paper.
\end{abstract}

Key words: anaerobes, extremophiles, chirality, Stickland reaction, inhibition of growth, Alterenantiomeric Microbial Communities, $D$-amino acids, $L$-sugars,

\section{INTRODUCTION}

It is well known that in living organisms, amino acids are genetically encoded and exhibit chirality. Except for glycine, which is symmetric, all protein amino acids are the Levorotary $(L-)$ enantiomer and all sugars in DNA, RNA and other life-critical biomolecules are the Dextrorotary $(D-)$ enantiomer. This is essential to permit for correct folding of proteins and enzymes into domains and pleated sheets and coiling of RNA and DNA into right handed helices. Conversely, amino acids, sugars and other molecules that exhibit opposite chirality are always formed by abiotic mechanisms as essentially racaemic mixtures $(D / L=1)$, even though some processes can result in a slight enantiomeric excesses. For this reason, the homochirality of biomolecules is considered a hallmark of life.

In 2000, while working on microbial extremophiles at the NASA/MSFC Astrobiology Laboratory we found some bacterial strains could grow on alternate chirality enantiomers. The type strain of our new genus and species Anaerovirgula multivorans was able to grow on $L$-arabinose, but it was unable to utilize $D$-arabinose. ${ }^{1}$ After discussions with Paul Davies concerning methods to search for a possible "Shadow Biosphere", we started checking our anaerobic extremophilics to determine their ability to grow on alternate enantiomers of sugars and amino acids. ${ }^{2}$ Our initial results of this study were presented in $2004 .^{3}$ We have continues this research and performed additional tests on already published species of extremophilic bacteria. Here we present new data on the recent experiments performed on pure and enrichment cultures. The tests conducted on pure cultures of extremophilic strains were:

- Determination of the ability to grow on alternate chirality enantiomers among different groups of extremophilic microorganisms

- Stickland reaction with alternate enantiomers

- Inhibition of growth by alternate enantiomers.

For enrichment cultures we performed tests for growth ability on alternate chirality mixtures. Samples collected from extreme ecosystems and containing viable microbial cells (based on microscopy) were: 
- Sediments from Owens Lake (California);

- Chena Hot Spring (Alaska);

- $\quad$ Sediments from Lake Untersee (Antarctica);

- Samples from Kverkfjol Glacier (Iceland);

- Mud samples from fumaroles (Iceland).

All samples were injected in anaerobic media, and cultivated at correspondent regimes of temperature, $\mathrm{pH}$, and salinity (according to in-situ environmental conditions).

Historically our knowledge about the metabolism of living organisms starts from chemoorganoheterotrophic type, later photosynthesis for plants and algae was discovered, and most amazing type was described by Sergey Winogradsky in 1895 - chemolithoautotrophic, which at first was shown as a nitrogen-fixation process in the free-living species Clostridium pasteurianum. Here we will discuss bacterial metabolic activity in the context of chemo-organo-hetero-trophy, since chirality is a feature exclusively of organic molecules.

Horizontal Gene Transfer (HGT) is a very important force driving the evolution of prokaryotic species, since it allows the acquisition of new genes that can facilitate the adaptation to different environments and conditions. It will be mistake to exclude the participation of this process during the evolution of microorganisms carrying the feature to metabolize opposite chirality enantiomers. The availability of metagenomic sequencing of such microorganisms can help to understand how HGT relates to community composition and environmental factors. Nevertheless, metagenomic sequences have particular characteristics that are not especially appropriate for the study of HGT, mainly their short length and their unknown origin. According to Tamames and Mirathe the extent of HGT is influenced by the physical and ecological features of the environment. ${ }^{4}$

Metagenomic analyses of natural biological communities are revolutionizing our understanding of the diversity, function, and inter-relationships among organisms in diverse ecological niches. ${ }^{5}$ The rapid advancements in metagenomics are largely due to the technical and analytical methods developed from high throughput platforms for cloning, DNA sequencing, robotics, high-density microarrays, 2D-gel electrophoresis, and mass spectrometry as well as associated bioinformatics software. Of special notes are the recent developments in single cell isolation, whole-genome amplification, pyrosequencing, and database warehousing and the potential impact of these developments on our future understandings of microbial communities in nature. It is quite possible that a metagenomic analyses is the key for the understanding of origin and distribution of the genetic units responsible for alternate enantiomers to be utilized.

Microbes are important for the ecosystem of planet Earth, playing a very important role in maintaining our global environment. Despite of the obvious importance of microbes, very little is known of their diversity, how many species are present in the environment, and the ecological function of each individual species. Until recently, there were no appropriate techniques available to answer these important questions. The vast majority of these microorganisms cannot be cultured in the laboratory and so were not amenable to study by the methods that had proved so successful with known microorganisms throughout the 20th century. It was only with the development of high-throughput technology to analyze and sequence DNA from the natural environment that information began to accumulate that demonstrated the exceptional diversity of microbes. ${ }^{6}$

At the present time the higher taxonomic groups within prokaryotes are mainly distinguished on the basis of their branching in phylogenetic trees. In most cases, no molecular, biochemical or physiological characteristics are known that universally combine the species from these groups. Phylogenetic analyses of genome sequences could be used for directed discoveries of novel molecular characteristics that are specific for different groups of bacteria and archaea and provide more precise characteristics for identifying and circumscribing these groups of microbes in clear molecular terms and for understanding their evolution. Perhaps the finding of the distribution of the capacity to metabolize alternate chirality enantiomers among prokaryotes will provide some outline on the origin of this unique physiological feature. We still do not know if this capability to utilize alternate enantiomers is limited to unicellular prokaryotic organisms or is it also a characteristic to be found in multi-cellular highly organized life? The recently described disease $L$-arabinosuria, which resulted from a human pentose metabolism defect in the ability of a 18 month-old child to metabolize the $L$-arabinose present in her fruit diet triggers this question. ${ }^{7}$

Advances in genomics have had a great impact on the field of microbial ecology. Metagenomics in particular holds great promise for accessing and characterizing microbial communities. ${ }^{8}$ However, the high diversity and level of complexity present in microbial communities represent an obstacle to understanding these assemblages given the current approaches. The integration of microbial community structure with function, taking into account uncultured microbes in diverse environments, remains particularly challenging.

\section{EXPERIMENTS WITH SUGARLYTIC ANAEROBES}




\title{
2.1. Moderately thermophilic alkaliphilic anaerobes
}

The facultatively anaerobic Anoxybacillus puchshinoensis strain K1 is an obligately alkaliphilic and moderately thermophilic bacterium that uses exclusively sugars for growth. It is spore-forming and Gram-positive with rod-shape cells that sometimes form Y-shape branching (Fig. 1). In 2000, Pikuta et al. presented the detailed description of this organism. ${ }^{9}$ In the study of this bacterium we have checked the ability to use the following alternate chirality sugars for growth: L-glucose, L-arabinose, L-mannose, and L-fucose. Each sugar was added to anaerobic highly mineralized medium in concentration $2 \mathrm{~g} / \mathrm{l}$ separately after autoclaving. The $\mathrm{pH}$ of the medium was 9.0, salinity $1 \% \mathrm{NaCl}$, and an incubation was conducted at $+62{ }^{\circ} \mathrm{C}$. As positive controls we used cultures grown on: $\mathrm{D}$-glucose, D-arabinose, D-mannose, and D-fucose (each $2 \mathrm{~g} / \mathrm{l}$ ). Also we checked an inhibitory effect of $D$-amino acids on growth of strain $\mathrm{K} 1^{\mathrm{T}}$. The following combinations of substrates were prepared:
a) $D$-glucose (3 g/l) $+L$-glucose $(4 \mathrm{~g} / \mathrm{l})$
b) $D$-glucose $(3 \mathrm{~g} / \mathrm{l})+D$-proline $(4 \mathrm{~g} / \mathrm{l})$
c) $D$-glucose $(3 \mathrm{~g} / \mathrm{l})+L$-proline $(4 \mathrm{~g} / \mathrm{l})$
d) $D$-glucose $(3 \mathrm{~g} / \mathrm{l})+L$-lysine $(4 \mathrm{~g} / \mathrm{l})$
e) $D$-glucose $(3 \mathrm{~g} / \mathrm{l})+L$-serine $(4 \mathrm{~g} / \mathrm{l})$.

\begin{abstract}
Anoxybacillus puchshinoensis $\mathrm{K}^{\mathrm{T}}$ is an exclusively saccharolytic bacterium that does not require vitamin supplements in the growth medium. It was isolated from an organic-rich environment - cattle manure. The inhibitory effect of $D$ - amino acids, as well as $L$ - sugars for culture growth, was tested. The results showed no growth abilities on separate $L$-sugars added: No growth was observed on L-glucose, $L$ - arabinose, $L$-mannose, and Lfucose. Controls with $D$-glucose and $D$-mannose were positive. However, growth on $D$-glucose (3 g/l) $+L$ glucose $(4 \mathrm{~g} / \mathrm{l})$ showed a higher rate compared to the control on D-glucose. That means, $L$-glucose added had a stimulating effect (as an additional substrate) for the culture growth (see table 1).

The existence of the racemase-group of enzymes for this strain is highly possible.

Opposite chirality sugars and amino acids did not restrict the growth of strain $\mathrm{K} 1^{\mathrm{T}}$ : all tubes had noticeable optic density. Nevertheless, on $D$-glucose (3 g/l) $+D$-proline (4 $\mathrm{g} / \mathrm{l})$ the optic density was 2.5 times lower than on $D$ glucose (3 g/l) $+L$-proline (4 g/l). That means that opposite chirality amino acid does have an inhibitory effect on growth of the strain $K 1$.

The absence of Y-shape cells on the medium with D-amino acids added, also means that a specific inhibitory effect for bifurcate cell division had occurred.

Inhibition of growth for strain $\mathrm{K} 1$ was determined on $D$-serine with significantly prolonged lagphase. The chemical changes of the red-ox potential during injection of substrate here are excluded since the medium was controlled with resazurin added and heavily buffered carbonate/bicarbonate system. We found this effect very interesting and it definitely requires continued study.

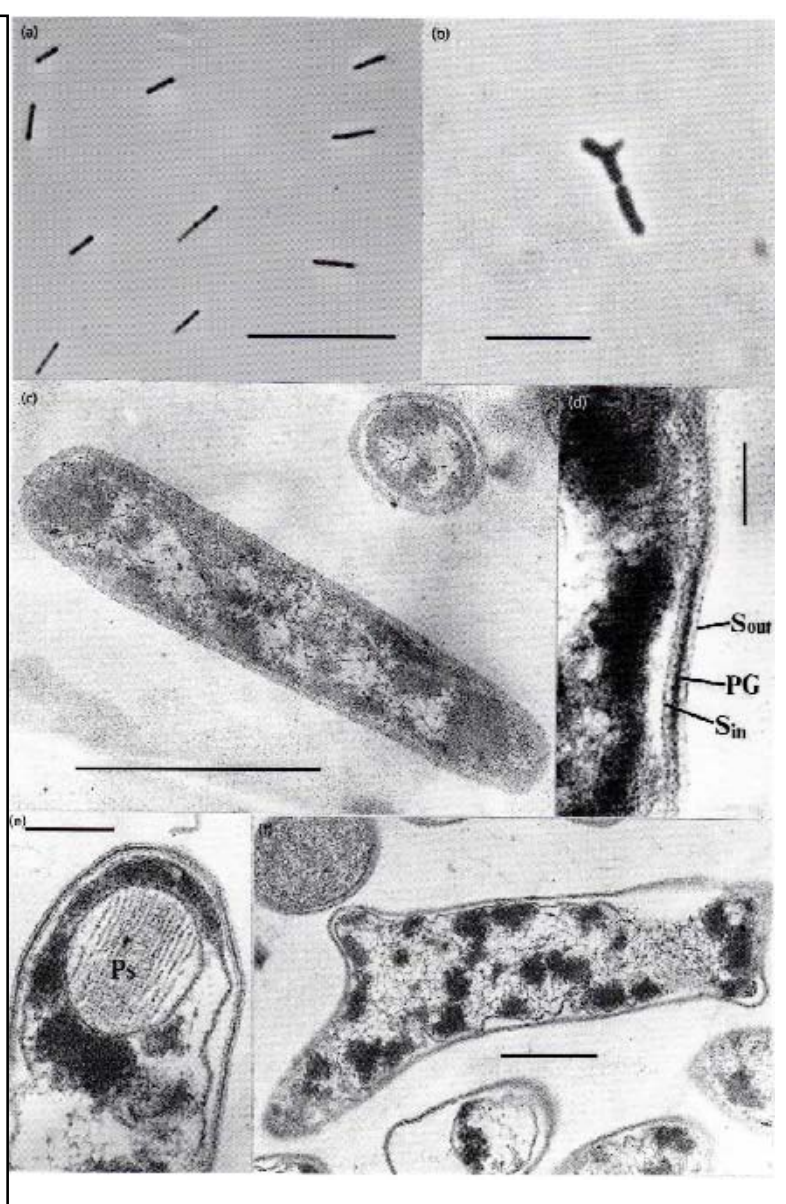

Fig. 1. Cell morphology of strain K1 presented by: straight rods (a,c); and Y-shaped cells (b,f).

The results of the tests conducted are presented in Table 1. Although the strain K1 did not grow on separate $L$-sugars, a very interesting observation was found for growth on the mixture of $D$ - and $L$ - glucose: the optic density was twice higher when compared with the positive control on $D$ - glucose. This may be explained by a purely chemical effect with higher reduced rate (Redox potential), or involvement of enzymes such as racemases in this microorganism.
\end{abstract}


Table 1. Cell number and morphological changes of strain K1 at cultivation on $D$ - amino acids.

\begin{tabular}{|c|c|c|c|c|}
\hline № & Growth substrate: & $\begin{array}{c}\text { Optic density } \\
\lambda=510 \mathrm{~nm}\end{array}$ & $\begin{array}{c}\text { Purpose of } \\
\text { measurement: }\end{array}$ & Cell morphology changes: \\
\hline 1. & $D$ - mannose & 0.057 & Control & 4-8 cells/field \\
\hline 2. & $D$-glucose & 0.143 & Control & Usual morphology (Fig. 1) \\
\hline 3. & $D$-glucose $+L$-glucose & 0.206 & Check out & $\begin{array}{l}\text { Cells are single, in pairs, Y- } \\
\text { cells occurs; } 60-70 \text { cells/field }\end{array}$ \\
\hline 4. & $D$-glucose $+D$-proline & 0.11 & Check out & $\begin{array}{l}\text { Cells are single, in pairs, no } \\
\text { Y-cells; } 10-15 \text { cells/field }\end{array}$ \\
\hline 5. & $D$-glucose $+L$-proline & 0.245 & Check out & $\begin{array}{l}\text { Long chains, cells curved, Y- } \\
\text { cells occurs; } 80-90 \text { cells/field }\end{array}$ \\
\hline 6. & $D$-glucose $+D$-lysine & 0.093 & Check out & $\begin{array}{l}\text { Cells single, in pairs, no Y- } \\
\text { cells; } 10 \text { cells/field }\end{array}$ \\
\hline 7. & $D$-glucose $+D$-serine & 0.005 & Check out & $\begin{array}{l}\text { Growth delayed: culture grew } \\
\text { up on second day }\end{array}$ \\
\hline
\end{tabular}

\subsection{Psychro- and alkali-tolerant neutrophilic culture}

Trichococcus patagoniensis PmagG1 ${ }^{\mathrm{T}}$ is psychrotolerant mesophilic, sugarlytic, facultatively anaerobic bacterium that was isolated from penguin (Spheniscus magelanicus) guano collected in South Patagonia, Chile during the Antarctica 2000 Expedition (Pikuta et al., 2006).$^{10}$ This bacterium is able to grow at $-5{ }^{\circ} \mathrm{C}$ by excreting exo-polysaccharides surrounding cell surface as a capsule. We performed several tests with this bacterium for growth on $D$ - and $L$-sugars. Positive results for growth were found for $D$-glucose (used here as a control), $D$ - and $L$ mannose, and $L$-arabinose. Negative growth was observed on: $L$ - and $D$-fucose, $D$-arabinose, $L$-glucose.

\section{TESTS WITH ORGANISMS PERFORMING THE STICKLAND REACTION}

After separate tests with individual $D$ - amino acids, as growth substrates for proteolytic bacterial strains, the idea to examine the Stickland reaction with $D$-amino acids was suggested. The Stickland reaction was discovered by Stickland in 1934 for the obligately anaerobic species Clostridium sporogenes. ${ }^{11}$ It was found for species containing cytochromes. The purpose of these cytochromes was unknown at that time, since for clostridia the only metabolic consumption of substrates was described through fermentation, which does not require the involvement of the respiratory chain with cytochromes. During investigation of the metabolic characteristics in this bacterium it was found that some pairs of amino acids could play the function of electron donors and others perform the function of electron acceptors at the same time, and as a result, support growth of the culture. Later the described reaction received the name of the first investigator - Stickland Reaction. This reaction belongs to the category of anaerobic respiration, and it also could be categorized as a two-substrate fermentation process. Not all amino acids could provide this unique reaction. The following amino acid pairs could be used for providing energy:

$L$-proline $+L$-alanine,

$L$-proline $+L$-valine,

$L$-proline $+L$-isoleucine,

$L$-proline $+L$-leucine,

$L$-tryptophan $+L$-valine,

glycine $+L$-alanine,

glycine $+L$-valine,

glycine $+L$-leucine,

glycine + L-isoleucine.

During the Stickland reaction one amino acid is reduced (such as glycine or proline) and the other one is oxidatively decarboxylated and deaminated (the branched chain and the aromatic amino acids, arginine, ornithine, 
threonine, or serine). In the classical Stickland reaction, glycine serves as the electron acceptor, and glycine is reduced to acetate and ammonia - a process that is coupled to ATP synthesis by substrate level phosphorylation. Acetyl phosphate here is an intermediate, and ATP is synthesized in the acetate kinase reaction. By observation of culture growth on Stickland reaction substrates the optic density of cells never yields significant biomass $(\lambda 510 \mathrm{~nm}$ $\leq 0.03$; or $0.9 \times 10^{7}$ cells $/ \mathrm{ml}$ ), but it supports growth in consequent transfers. The reaction could be explained as a survival strategy in the process of the evolution for life during periods with a limitation of energy sources.

So, the purpose of our experiments with the Stickland reaction conducted on opposite chirality enantiomers of amino acids was to find out if the reaction itself could be an absolute alternate and composed of two $D$ - amino acids or, when at least one of them has an alternate chirality enantiomer (partially alternate). For this reason we have prepared anaerobic media with the following combinations of amino acids:

1.

$D$-pro $+D$-val $\quad$ - absolute alternate chirality reaction

$D$-pro $+L$-val $\quad$ - partially alternate chirality reaction

$L$-pro $+D$-val - partially alternate chirality reaction

Control reaction was: $L$-pro $+L$-val

2.

$D$-pro $+D$-ala - absolute alternate chirality reaction

$D$-pro $+L$-ala $\quad$ - partially alternate chirality reaction

Control reaction was: $L$-pro $+L$-ala

- partially alternate chirality reaction

3.

$D$-pro $+D$-leu - absolute alternate chirality reaction

$D$-pro $+L$-leu - partially alternate chirality reaction

$L$-pro $+D$-leu - partially alternate chirality reaction

Control reaction was: $L$-pro $+L$-leu

4.

$$
\begin{array}{ll}
\text { gly }+D \text {-val } & \text { - absolute alternate chirality reaction } \\
\text { gly }+D \text {-his } & \text { - absolute alternate chirality reaction } \\
\text { gly }+D \text {-ala } & \text { - absolute alternate chirality reaction } \\
\text { gly }+D \text {-leu } & \text { - absolute alternate chirality reaction }
\end{array}
$$

5.

$$
\begin{array}{ll}
D \text {-try }+D \text {-val } & \text { - absolute alternate chirality reaction } \\
D \text {-try }+L \text {-val } & \text { - partially alternate chirality reaction } \\
L \text {-try }+D \text {-val } & \text { - partially alternate chirality reaction }
\end{array}
$$

The only asymmetric amino acid here is glycine, which belongs to mono-amino and mono-carbonic group, and also, is unique in that it is the only protein amino acid that does not exhibit chirality. Alanine, leucine, and valine are all belong to hydrophobic-aliphatic group of amino acids. Histidine is basic and hetero-cyclic amino acid. Proline also belongs to the hetero-cyclic amino acids and to a unique group.

Organisms for checking these substrate combinations were mesophilic and obligately alkaliphilic anaerobes:

1) saccharolytic bacterium Anaerovirgula multivorans strain $S C A^{T}$,

2) texclusively proteolytic bacteria Tindallia californiensis str. $\mathrm{APO}^{\mathrm{T}}$ \& Tindallia magadiensis str. Z-7934 ${ }^{\mathrm{T}}$.

Two other organisms with features of proteolytic and saccharolytic bacteria strain FTR1 ${ }^{\mathrm{T}}$ (Carnobacterium pleistocenium) and strain $\mathrm{SCA}^{\mathrm{T}}$ (Anaerovirgula multivorans) were also checked for the Stickland reaction.

\section{EXPERIMENTS WITH STRICTLY PROTEOLYTIC ANAEROBES}

\subsection{Alkaliphilic mesophilic cultures}

Tindallia californiensis $\mathbf{A P O}^{\mathrm{T}}$ was our first proteolytic bacterium in Astrobiology Laboratory collection at NASA/NSSTC. This mesophilic and obligately alkaliphilic bacterium was isolated from anaerobic sediment sample of Mono Lake in California. This strain was described in detail in Extremophiles, ${ }^{12}$ where all possible combinations of $L$-amino acids were presented for the description of the Stickland reaction. In this study we have checked growth of this bacterium on separate $D$-amino acids and on pairs of $D$-amino acids (all possible combinations were discussed in previous chapter). 
The Stickland reaction was positive for strain APO for all tree possible combinations (absolute and partially alternate chirality) with: $D$-proline $+D$-alanine, $D$-proline $+L$-alanine, and $L$-proline $+D$-alanine. Positive control here was the combination $L$-proline $+L$-alanine.

The Stickland reaction was also positive for Tindallia californiensis strain APO on the media with: glycine $+D$-alanine and glycine $+D$-leucine.

Positive controls here were: glycine $+L$-alanine and glycine $+L$-leucine.

The next combination of substrates for the Stickland reaction of strain APO showed the following results: Positive growth was on combinations: $D$-tryptophan $+D$-valine, and $L$-tryptophan $+D$-valine.

Negative growth was on combination: $D$-tryptophan $+L$-valine.

Positive control was on: $L$-tryptophan $+L$-valine.

Strain $\mathrm{APO}^{\mathrm{T}}$ also grew on separate alternate chirality amino acids:

$D$-arginine, $D$-lysine, and $D$-serine.

For all these amino acids positive controls used $L$-enantiomers. The growth of strain APO on $D$-serine (Fig. 2) was limited by massive spore-formation, which is very rare for strain APO (this species was described as oligospore-forming, which means spore-formation is rarely observed and difficult to produce).

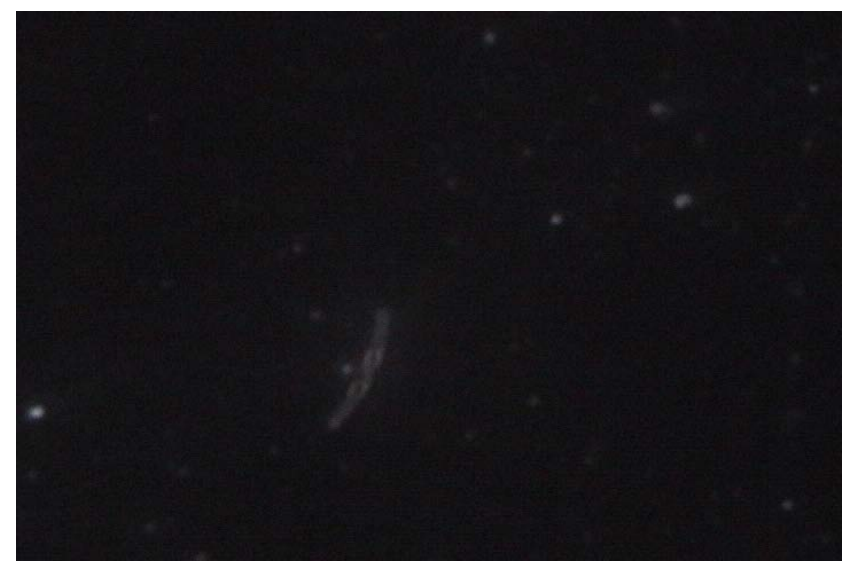

Fig. 2. Strain APO grown on $D$-serine: two dividing cells are in the process of spore-formation.

Another interesting observation was made for strain APO grown on $L$-histidine (3 g/l). This growth was inhibited by $D$-proline (4 g/l).

Growth without morphological changes was determined on:

1. Glycine $+D$-alanine; Cells were motile, dividing, no spores.

Optic density of positive control on glycine $+L$-alanine was half the optic density on glycine $+D$-alanine.

2. $D$-proline $+D$-alanine (6-8 cells per field, cells are motile and dividing).

3. $L$-proline $+D$-alanine (8 cells per field; short chains, motile, no spores).

4. $D$-proline $+L$-alanine (6-7 cells per field, short chains, motile, dividing.

5. $D$-arginine (6 cells per field, cells are motile dividing).

6. $D$-tryptophan $+D$-valine (weak growth)

7. Positive controls: $L$-proline $+L$-alanine and $L$-tryptophan $+L$-valine, $L$-serine.

\subsection{Experiments with Tindallia magadiensis Z-7934 ${ }^{\mathrm{T}}$.}

Strain Z-7934 ${ }^{\mathrm{T}}$ is the type strain of first species (T. magadiensis.) described in the genus Tindallia. This organism was isolated from anaerobic sediments of Lake Magadi in Kenya, Africa. The standard description presented in the original article ${ }^{13}$ provided data only for $L$-amino acids. For comparison we had checked this strain for the Stickland reaction with pairs and growth on separate $D$-amino acids.

Tests with separate $D$-amino acids ( $D$-serine, $D$-lysine, $D$-histidine, and $D$-aspartic acid) were conducted to check the growth ability of strain Z-7934. It was found that growth of strain Z-7934on $D$-histidine demonstrated a significantly higher rate of increase in optic density than on $L$-histidine. This species was described with weak 
growth ability on $L$-histidine (Kevbrin et al., 1996). ${ }^{13}$ Good growth also occurred on $D$-lysine (with positive control on $L$-lysine). This strain also had weak growth on $D$-aspartic acid. There was no growth on $D$-serine.

Stickland reactions for strain Z-7934 were positive for: $D$-proline $+D$-valine; $D$-proline $+L$-valine. The combination of $L$-proline $+D$-valine gave negative result. As positive control here was $L$-proline $+L$-valine.

No growth was found for strain Z-7934 on $D$-proline $+D$-leucine. But combinations with $D$-proline $+L$ leucine and $L$-proline $+D$-leucine were positive. Also positive tests were with combinations: $D$-proline $+D$-alanine, $D$-proline $+L$-alanine, and $L$-proline $+D$-alanine.

The growth of strain Z-7934 on $D$-arginine, $D$-glutamine and $D$-threonine - was not checked.

The Stickland reaction on combinations with glycine was positive for strain Z-7934 on:

glycine $+D$-valine, glycine $+D$-alanine, and glycine $+D$-leucine.

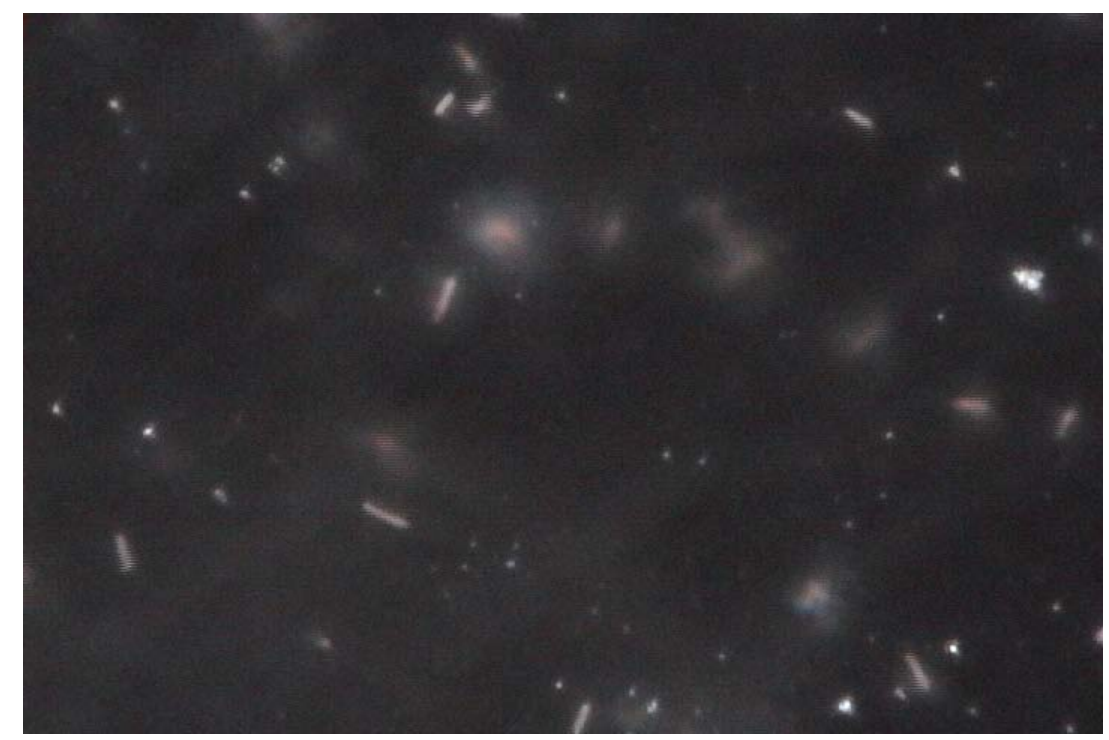

Fig. 3. Culture of Tindallia magadiensis Z-7934 ${ }^{\mathrm{T}}$ grown on $D$-histidine.

\section{EXPERIMENTS WITH BACTERIA COMBINING PROTEO- AND SACCHAROLYTIC METABOLISMS}

\subsection{Mesophilic halo-alkaliphilic anaerobes}

Anaerovirgula multivorans SCA was the first in our laboratory bacterial strain, on which we found the ability to grow on opposite chirality sugars (Pikuta et al., 2006). ${ }^{3}$ This bacterium is able to use a wide spectrum of growth substrates, including sugars, proteolysis products, and some organic acids. For this reason strain SCA was used for experiments with both sugars and amino acids.

The experimental work was conducted on the mesophilic obligately halo-alkaliphilc anaerobe Anaerovirgula multivorans SCA to implement the following tests: 
- Stickland reaction on: $D$ - proline $+L$ isoleucine and $D$ - proline $+L$ - luecine (Positive controls: $L$ - proline $+L$ isoleucine and $L$ - proline $+L$ - leucine)

- $\quad$ Compare growth on $L$ - and $D$ - ribose, $L$ $\& D$ - mannose

- Growth on $D$ - \& $L$-arabinose. (Why it grew on $L$-arabinose and does not on $D$ arabinose?)

- Inhibition by $D$-proline on $D$-glucose (with positive control $L$ - proline $+D$ glucose)

- Inhibition by $D$ - proline on $L$ - ribose and $L$ - arabinose.

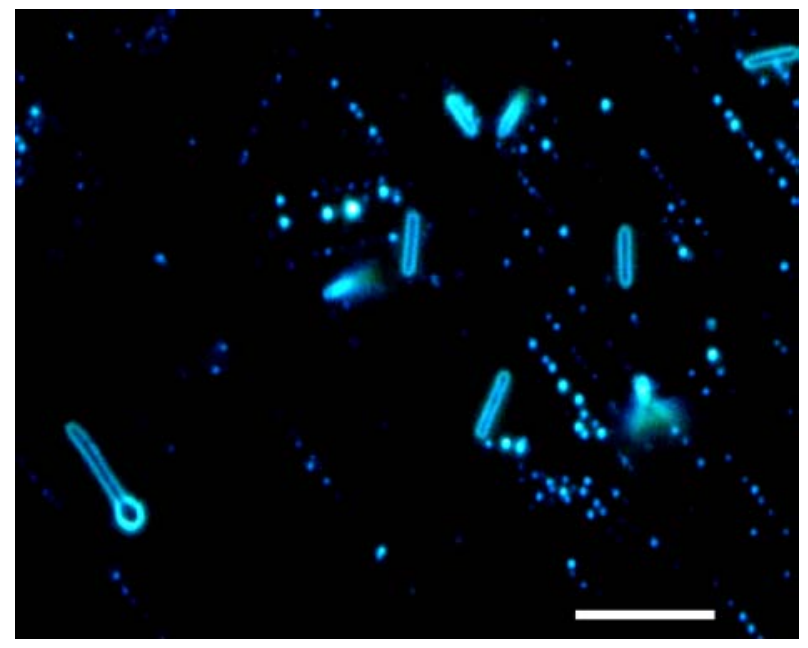

Fig. 4. Cells of Anaerovirgula multivorans $\mathrm{SCA}^{\mathrm{T}}$. Bar $=10 \mu \mathrm{m}$.

The experiments with mesophilic alkaliphilic culture SCA resulted in the following:

- $\quad$ No growth was determined on $L$-glucose (growth on $D$-glucose was a positive control).

- $\quad$ No growth was found on $D$-fucose and $L$-fucose.

- Growth on $D$ - ribose had high optic density in all three transfers.

- $\quad L$-ribose was not checked.

- $\quad$ Strain $\mathrm{SCA}^{\mathrm{T}}$ grew well on $D$-mannose, but was not able to grow on $L$-mannose.

- As shown previously, the strain $\mathrm{SCA}^{\mathrm{T}}$ was not able to grow on $D$-arabinose, but grew well on $L$-arabinose.

- The performed tests showed no evidence for the inhibitory effect by $D$-proline on the culture grown on $D$ glucose. Contrarily, significant stimulating growth effect was determined: the cell number was higher than in control, cells did not form spores and had a tendency to form short motile chains.

Since strain $\mathrm{SCA}^{\mathrm{T}}$ was not able to grow on separate amino acids $L$-proline and $L$-leucine and tests for the Stickland reaction were suggested to be performed. Results of experiments demonstrated that the combination of $L$-proline $+D$-leucine did not support growth, but medium containing $D$-proline $+L$-leucine showed noticeable optic density. As was determined later, the culture was able to grow on $D$-proline alone.

\subsection{Psychro- and alkali-tolerant neutrophilic culture}

Several tests were performed on psychrotolerant bacterium Carnobacterium pleistocenium FTR $1^{\mathrm{T}}$ which was isolated from an ice lens of a frozen thermokarst pond within the CRREL Fox Permafrost Tunnel in Alaska ${ }^{.14}$ This facultatively anaerobic, gram-positive and non spore-forming bacterium has alkalitolerant nature and could grow at $\mathrm{pH}$ 9.5. It also tolerates multiple freeze/ thaw cycles. In this work we checked growth ability of the strain $\mathrm{FTR} 1^{\mathrm{T}}$ to grow on $D$ - and $L$ - sugars and amino acids. Results were:

- Strain FTR1 $1^{\mathrm{T}}$ was able to grow on the following substrates: $D$-glucose, $D$ - and $L$-mannose, $D$ - and $L$ arabinose, $L$-lysine, $D$-serine (+), $L$-serine (+), $D$-proline, $L$-histidine.

- $\quad$ No growth was determined for: $L$-glucose, $L$ - and $D$-fucose, $D$-lysine, $D$-histidine, $L$-arginine, $L$-aspartic acid, $L$-proline, $L$ - and $D$ - aspartic acid.

\section{Conclusions about strain FTR1 ${ }^{\mathrm{T}}$ :}

- The strain could grow on opposite chirality enantiomers (both sugars and amino acids). This fact agrees with the ecological where the microorganism was isolation. The sample was collected from Pleistocene permafrost $\sim 32,000$ years old. The organic material of this sample had probably experienced changes of chirality by racemization during natural geochemical processes. That also provides evidence for the active metabolism of this bacterium functioning at low temperatures in constant adaptation to surrounded substrate changes.

- This organism did not just survive and wait to be unfrozen, but was definitely functional within the ice lens and had time for long-term adaptation to new substrate. 


\section{EXPERIMENTS WITH ENRICHMENT CULTURES}

\subsection{Sugarlytic moderately thermophilic culture}

The ability to grow on alternate chirality enantiomers was also checked for enrichment cultures of anaerobic termophiles. The samples of hot water and mud were collected in fumaroles in Iceland during the 2009 BBC sponsored Expedition. Mixture of water and mud slurry from the fumaroles ( $0.3 \mathrm{~g}$ ) was injected in Hungate tubes with mineral medium containing mixture of $L$-sugars ( $L$-glucose, $L$-fucose, $L$-mannose, and $L$-arabinose). Incubation at $60{ }^{\circ} \mathrm{C}$ during $30 \mathrm{~h}$ resulted in an enrichment culture composed of irregular coccoid shape cells (the dominant morphotypes) and slightly curved rods. Consequently, three transfers revealed exclusively coccoidal cell cultures; the ulture had a strong smell of hydrogen sulfide and in the phase-contrast microscope showed actively motile coccoid cells. No other sources of energy ( $D$-sugars or $L$-amino acids) were added in the medium.

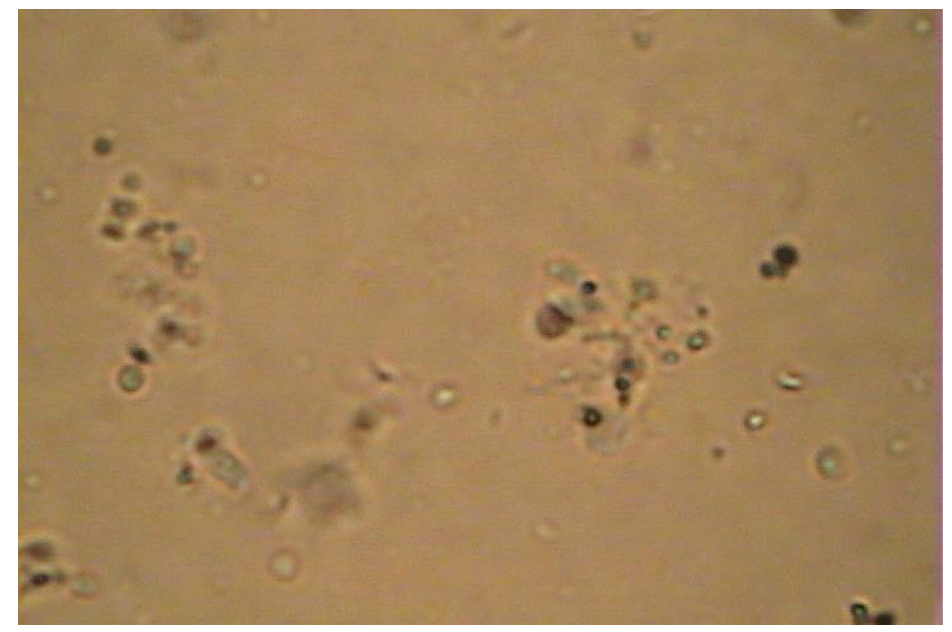

Fig. 5. Enrichment culture from Iceland fumarole grown up on mixture of $L$-sugars.

\subsection{Proteolytic cultures}

Enrichment cultures from the Owens lake sample had positive growth on both groups of substrates: $D$ amino acids and $L$-sugars. The mixture for $D$-amino acids contained: proline, histidine, lysine, and serine. Third transfers showed more than one phenotype that allows us to advance the concept of "alternate chirality communities" in the microbial world, rather than just separate species.

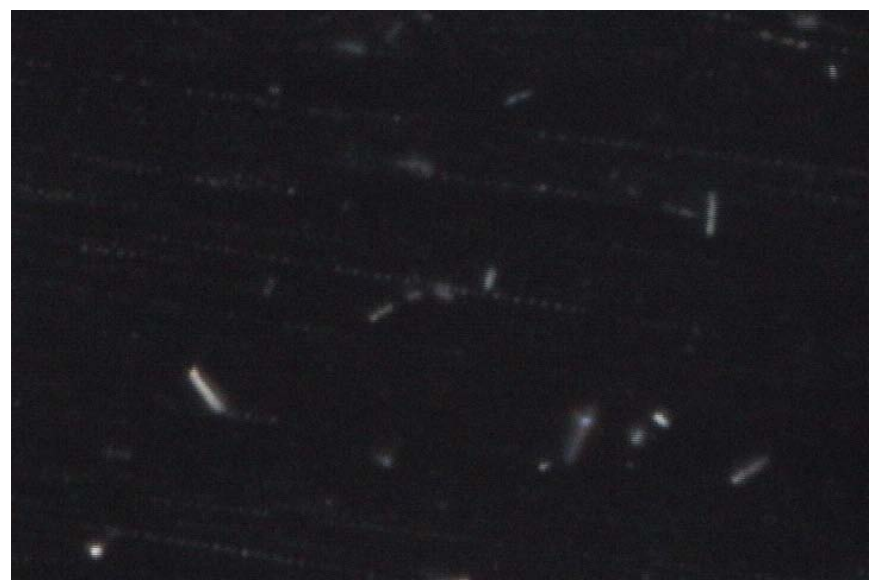

Fig. 6. Enrichment culture obtained on the mixture of $D$-proline, $D$-histidine, $D$-lysine, and $D$-serine. 


\section{DISCUSSION}

The hypothetical term "Shadow Biosphere" was introduced comparatively recently. Philosophy professor Carol Cleland first coined the term "shadow biosphere" in 2005, while heading an investigation into the definition of "life" at the NASA-funded CU-Boulder Center for Astrobiology. Professor Cleland described Earth's shadow biosphere as: “... a microbial biosphere that is so chemically and molecularly different from life as we know it that it wouldn't be in direct competition with familiar life; familiar life couldn't metabolize it and it would occupy ecological niches that were under populated by familiar organisms. Models representing the "Shadow Biosphere" could be described by means of several types. The first represents organisms, the life-critical constitutional biomolecules of which are composed exclusively of $D$-amino acids and $L$-sugars, and their genome consists exclusively of $L$-sugars in the nucleotides. Such organisms if they exist cannot be detected by modern molecular methods that are based on application of polymerase technique.

Estimation of biogeochemical processes never led to conclusive results for global measurements of biogeocycles, since many new types of metabolic pathways were discovered for de novo described microbial species during the calculations. Accounting for the possibility of new acceptors and donors of electrons and unexpected biochemical pathways had required recalculations and analysis from the beginning for every ecosystem. So, only approximate data for biogeochemical activity now exists in science. The involvement of the undiscovered "Shadow Biosphere" in biogeochemical cycles was never a subject for serious consideration.

In this article we presented data not for absolute "Shadow Biosphere" microorganisms, but instead for normal terrestrial extremophilic species which have the abilities to utilize enantiomers of different chirality of some amino acids and sugars at the same time. The processes of mineralization of alternate chirality enantiomers for these known bacterial species are not estimated and therefore could contribute in "Shadow Biosphere" activity as competing for diverse substrates.

Presently in microbiology there is no official classification of prokaryotes, and furthermore, for the higher taxa there is no official nomenclature: the rules of the International Code of Nomenclature of Prokaryotes do not cover taxa above the rank of class. The most commonly accepted division of the prokaryotes into two "subkingdoms" or "domains" (Bacteria and Archaea) and the classification of their species with validly published names in respectively 27 and 2 "phyla" or "divisions" (as of November 2009) is primarily based on 16S rRNA sequence comparisons. ${ }^{15}$ This type of classification was adopted in the latest edition of Bergey's Manual of Systematic Bacteriology. Alternate classifications have been proposed as well, based for example on the structure of the cell wall. Some 16S rRNA sequence-based phyla unites prokaryotes of similar physiological properties (for example Cyanobacteria, Chlorobi, Thermotogae); others (Euryarchaeota, Proteobacteria, Flavobacteria) contain organisms with highly disparate lifestyles. Some phyla based on deep 16S rRNA lineages are currently represented by one or a few species only. Environmental genomics/metagenomics approaches suggest the existence of many more phyla based on the deep lineages of 16S rRNA gene sequences recovered. Isolation of living cultures of the microorganisms harboring these sequences, and the study of their properties are the major puzzles for microbiologists today. ${ }^{9}$

According to the current standards of Bacterial Taxonomy and Systematics, the species of all known microbial communities have been characterized only with traditional substrates: $L$-amino acids and $D$-sugars but not (or very rarely and only partially) for alternate chirality enantiomers. This means that virtually all microbial species have been ignored for testing their ability to grow on an alternate chirality diet. At present, among Prokaryotes there are about 10,000 validly published names in the category of species. According to Professor Euzeby, there are about 9,841 validly published names in the category species (of which 31 are later homotypic synonyms cited in the Approved Lists of Bacterial Names, 1191 are new combinations, 11 are nomina nova, about 265 are considered as later heterotypic synonyms, and 67 are illegitimate).

See Number of published names (http://www.bacterio.cict.fr/number.html).

Some not validly published bacterial names are cited in the file http://www.bacterio.cict.fr/nonvalid.html

It should be pointed out that this number of described bacterial species is an effort of all microbiologists for the period of time $\sim 150$ years. So, how many of these species do have ability to metabolize alternate chirality sugars and amino acids? The Photo- and litho-trophic species should be excluded from this number (less than $2 \%$ ); here only microorganisms utilizing organic molecules with $L$-or $D$ - enantiomers are considered. How much time it will take to check this characteristic? It is probably not realistic for one generation. In the literature, there are only a few records of the utilization of alternate chirality enantiomers since the 1960's.

Now we know that several modern neutrophilic (by pH) and mesophilic (by temperature) bacteria do have this ability as well as some hyperthermophilic sulfur-reducing archaea. Already from these observations, we can say that 
the gene that makes this possible, probably existed from beginning of life on Earth, and it is still here in a modern physico-chemical environment. This raises the following questions::

- Why do not all microorganisms have this feature? The majority apparently do not.

- Was this gene used in the beginning of the biosphere formation and no longer needed in the latest evolutionary environment - is it atavism - an evolutionary throwback?

- Could this gene have come from an extraterrestrial biological system that was injected at a certain period of Earth's bio-evolution and is still present in our genome? If so - could we determine when it happened? Or are these genes continuing to arrive with the rain of cometary debris and meteorites?

We know the special group of enzymes, racemases, is the key for the ability to use alternate enantiomers for energetic needs within cell metabolism. We know also that the genetic information encoding this group of enzymes could be located within RNA chromosome, or in additional and replaceable genetic units - plasmids.

To answer the question: "How old is this gene and how stable is it?"-we probably will need two generations. It could be a wonderful topic for a future astrobiology $\mathrm{PhD}$ dissertation - An investigation/screening of the pheno-genotypic features of all microbial species, or at least, central groups.

If we will consider the point of view that life on Earth evolved in the vector $\rightarrow$ from acidic and hot loving cells $\rightarrow$ to cold-loving/or -tolerant \&alkaline-increasing mineral salinity biota (Pikuta et al., 2007) ${ }^{16}$ and accept the possibility for the appearance of alternate chirality molecules already on the primordial Earth, then it should be reflected and memorized within genomic or chromosomal DNA/RNA material (group \# 1 on fig.1).

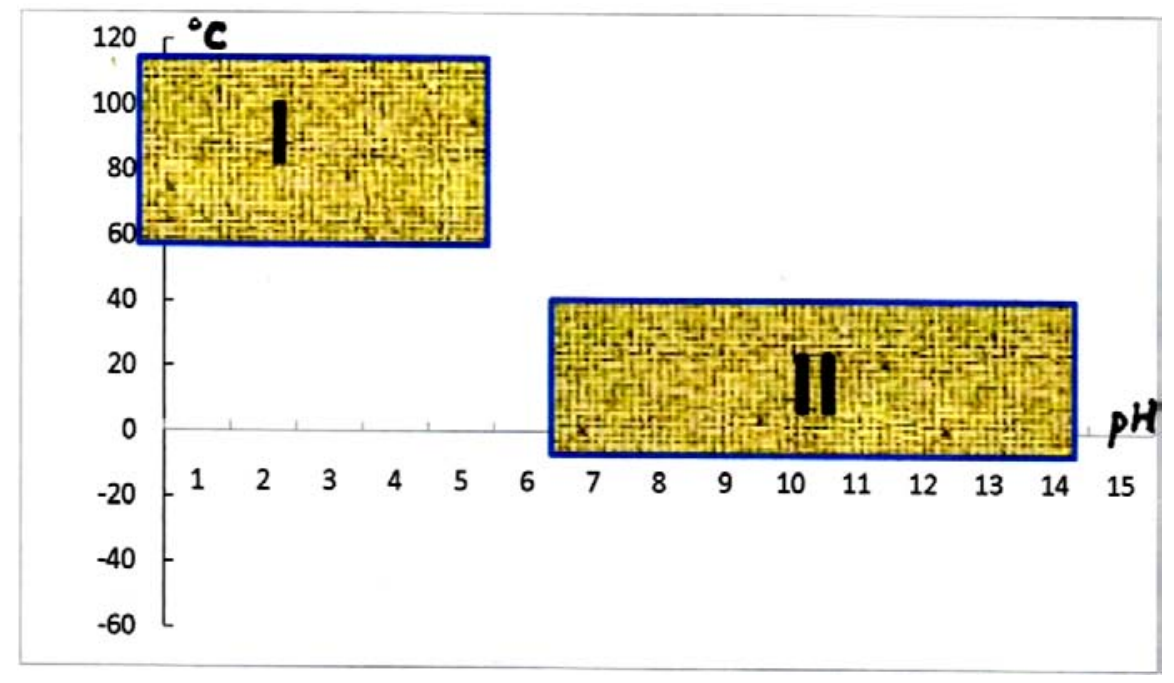

Fig. 7. Distribution of microbial species in matrix $\mathrm{pH} /$ Temperature, ${ }^{\circ} \mathrm{C}$; $\mathrm{I}-$ is covered by relic termophilic acidophiles, and II - by evolutionary latest neutro-/alkaliphiles.

- Alternatively, if the capacity for the utilization of alternate chirality enantiomers will be found in modern or the latest developed bacterial/archaeal cells (group \#2 on Fig. 7) then we are looking for the conclusion that this feature more likely has an extraterrestrial origin and these genetic units are imported from the Cosmos, and could have an unstable and substitutable character (i.e., plasmids, transposons, viruses, etc.)

- Where should one start looking for this gene among the entire biodiversity of Prokaryotes?

- Logically, it may be more productive to apply not a phylogenetic but an eco-physiological or evolutionary environmental physico-chemical approach.

- The central taxa of major groups of extremophilic microorganisms beginning from acidophilic thermophiles to alkali-heutrophilic mesophiles/psychrophiles should be screened on the genetic marker if such is to be found. 


\section{CONCLUSIONS}

Here we presented the results of experiments conducted on three physiologically different groups of microorganisms: moderately thermophilic alkaliphiles, mesophilic halo-alkaliphiles, and psychrotolerant neutrophiles.

- We found that moderately thermophilic strain $\mathrm{K} 1^{\mathrm{T}}$ did not show the direct ability to use $L$-sugars for growth, but they did grow better on a mixture of $L$ - and $D$-sugars.

- An onhibiting growth effect of $D$-amino acids, particularly D-serine, occurs for this organism.

All checked halo-alkaliphilic mesophiles (isolated from alkaline soda lakes) in our laboratory collection (strains $\mathrm{SCA}^{\mathrm{T}}, \mathrm{APO}^{\mathrm{T}}, \mathrm{Z}-7934^{\mathrm{T}}$, and $\mathrm{BK} 1$ ) demonstrated the ability to use alternate chirality enantiomers (on sugars as well as on amino acids).

- It is interesting that Anaerovirgula multivorans $\mathrm{SCA}^{\mathrm{T}}$ was found to be able to grow on $D$-proline and unable to grow on $L$-proline.

- Also it is puzzling why this strain grew well on $L$-arabinose but did not on $D$-arabinose?

- It was also shown that Tindallia californiensis strain $\mathrm{APO}^{\mathrm{T}}$ does have an ability to use alternate chirality amino acids alone as well as in pairs for the Stickland reaction. It grew well on $D$ arginine, glycine $+D$-alanine, $D$-tryptophane $+D$-valine, $D$-proline $+D$-alanine, etc...

Psychro-active alkali-tolerant neutrophilic cultures also demonstrated the ability to use alternate chirality enantiomers of sugars and amino acids.

- Psychrotolerant strain PmagG, which is able to grow at $-5{ }^{\circ} \mathrm{C}$, was also found to be able to grow on $D$ and $L$-mannose. It also grew on $L$-arabinose but was unable to grow on $D$-enantiomer of this sugar (just like in case of alkaliphilic, mesophilic, spore-forming strain $\mathrm{SCA}^{\mathrm{T}}$ ).

- Finally, psychrotolerant strain FTR1 $1^{\mathrm{T}}$ isolated from the Pleistocene sample demonstrated good growth on both $D$ - and $L$-enantiomers of mannose and arabinose.

- It also grew on $D$-serine with biomass growth twice better than on $L$-serine.

- Absolute loss of the ability to grow on $L$-proline but positive growth on $D$-proline makes strain FTR1 $1^{\mathrm{T}}$ a prime target for future investigations.

After all checkups of extremophilic anaerobic cultures and work with enrichment cultures obtained from different environmental samples we came to an absolutely unexpected conclusion:

- It is time to start the study of biogeo-ceonosises in entire complexity of a system from point of view racemization. In this article, we introduce the new term "alterenantiomeric microbial communities" for the designation of microbial communities that utilize alternate chirality molecules. "Alterenantiomeric Microbial Communities" do exist, and they are widely distributed geographically and waiting to be studied and/or reanalyzed.

\section{Acknowledgments}

We thank the administration of the National Space Sciences and Technologies Center and Von Broun Center for Science \& Innovation, Inc. and Delaware State University for providing funding support and facilities. Special thanks we express to Prof. Paul Davies (Arizona State University) for keeping interest in this field of research.

\section{REFERENCES}

[1] Pikuta, E.V., Itoh, T., Krader, P., Tang, J., Whitman, W.B. and R.B. Hoover. “Anaerovirgula multivorans gen. nov., sp. nov., a novel spore-forming, alkaliphilic anaerobe isolated from Owens Lake, California,” Int. J. Syst. Evol. Microbiol. 53, 2623-2629 (2006). 
[2] Davies, P.C.W., Benner, S.A., Cleland, C.E., Lineweaver, C.H., McKay, C.P. and F. Wolfe-Simon. "Signatures of a Shadow Biosphere,” Astrobiology 9, 241-249 (2009).

[3] Pikuta, E.V., Hoover, R.B., Klyce, B., Davies, P.C.W. and P. Davies. "Bacterial utilization of $L$-sugars and $D$ amino acids," Proc. SPIE, 6309, 63090A (2006).

[4] Tamames, J. and Mira, A. "Horizontal gene transfer in prokaryotic microbiomes," In: Metagenomics:Theory, Methods and Applications (Ed. D. Marco), Caister Academic Press 3, 39-53 (2008).

[5] Marco, D. (Ed.) “Metagenomics: Theory, Methods and Application,s”. Caister Academic Press, 1-212 (2010).

[6] Gupta, R. S. and Gao, B. "Recent Advances in Understanding Microbial Systematics,” In: Microbial Population Genetics (Ed: J. Xu) Caister Academic Press, 1-214 (2010)

[7] Okenhout, W., Groener, J. E., Verhoeven, N. M., Yin, C. and L. Laan, "L-Arabinosuria: A new defect in human pentose metabolism. Mol. Genet. Metab., 77, 80-85 (2002).

[8] Cubillos-Ruiz, A., Junca, H., Baena, S., Venegas, I. and Zambrano, M. M. "Beyond Metagenomics: Integration of Complementary Approaches for the Study of Microbial Communities” 1-96 (2009). http://www.slideshare.net/guest5368597/beyond-metagenomics-integration-of-complementary-approaches-for-thestudy-of-microbial-communities

[9] Pikuta, E.V., U. Mendrock, H. Hippe, Chuvilskaya N.A., Lysenko A.M., Suzina N., Nikitin D., Osipov G.A., Laurinavichius K. "Anoxybacillus pushchinensis gen. nov., sp. nov., a novel anaerobic alkaliphilic, moderately thermophilic bacterium from manure, and description of Anoxybacillus flavithermus comb. nov.” Int. J. Syst. Evol. Microbiol. 50, 2109-2117 (2000).

[10] Pikuta, E.V., Hoover, R.B., Bej, A.K., Marsic, D., Whitman, W.B., Krader, P.E. and J. Tang. “Trichococcus patagoniensis sp. nov., a facultative anaerobe that grows at $-5{ }^{\circ} \mathrm{C}$, isolated from penguin guano in Chilean Patagonia,” Int. J. Syst. Evol. Microbiol. 56, 2055-2062 (2006).

[11] Stickland, L.H. "Studies in the metabolism of the strict anaerobes (genus Clostridium). I. The chemical reaction by which Clostridium sporogenes obtains energy.” Biochem. J. 28, 1746-1759 (1934).

[12] Pikuta, E. V., Hoover, R. B., Bej, A. K., Marsic, D., Detkova, E. N., Whitman, W. B. and P. Krader “Tindallia californiensis sp. nov., a new anaerobic, haloalkaliphilic, spore-forming acetogen isolated from Mono Lake in California,” Extremophiles 7, 327-334 (2003).

[13] Kevbrin, V. V., Zhilina, T. N., Rainey, F. A., and G. A. Zavarzin "Tindallia magadii gen. nov., sp. nov.: an alkaliphilic anaerobic ammonifier from soda lake deposits,” Curr. Microbiol., 1998, 37, 94-100 (1998).

[14] Pikuta, E.V., Marsic, D., Bej, A.K., Tang, J., Krader, P., and Hoover, R.B. “Carnobacterium pleistocenium sp. nov., a novel psychrotolerant, facultative anaerobe isolated from permafrost of the Fox Tunnel in Alaska." Int. $J$. Syst. Evol. Microbiol., 55, 473-479 (2005).

[15] Oren, A. "The Phyla of Prokaryotes, Cultured and Uncultured,” In: Molecular Phylogeny of Microorganisms. (Ed(s): A. Oren and R. T. Papke) Caister Academic Press, 1, 225 (2010).

[16] Pikuta, E.V., Hoover, R.B. and J. Tang. "Microbial extremophiles at the limits of life," Critic. Rev. Microbiol. 33, 183-209 (2007). 